\title{
Annealing of Sputtered InSb Thin Film for III-V Semiconductor Devices Applications
}

\author{
Mohammad Awwad*, Ahmed Hafez ${ }^{\dagger}$, and Karam Sharshar ${ }^{\sharp}$
}

\begin{abstract}
In this paper, the effect of annealing temperature on Indium antimonide (InSb) thin films has been studied. It is proved that the increase in annealing temperature enhances the crystallinity of the InSb thin film. Thin films of InSb have been deposited on silicon and glass substrates by RF sputtering, then vacuum-annealed at 150, 300, 390 and $450{ }^{\circ} \mathrm{C}$. Differential scanning calorimeter (DSC) analysis indicates that InSb thin film crystallizes at $389{ }^{\circ} \mathrm{C}$ or above. X-ray diffraction (XRD) results show peaks at (111), (220) and (311) planes, which indicates the formation of crystalline grains. Fourier transform infrared (FTIR) spectroscopy indicates the decrease of optical transmittance upon increasing the annealing temperature, the analysis shows that InSb thin films have bandgaps between 0.233 and 0.241 e.v.
\end{abstract}

Keywords: InSb thin film, RF sputtering, annealing.

\section{Introduction}

Compound semiconductors play a very important role in the Optoelectronics field due to its direct bandgap structure and high mobility. Although the elemental semiconductors, particularly $\mathrm{Si}$, have been very useful for the development of microelectronics, the fundamental bandgap of these semiconductors is indirect. This implies that they emit light and/or electromagnetic waves very poorly. The most widely used compound semiconductors for optoelectronic device applications are III-V semiconductors [1].

Among the III-V binary semiconductors, indium antimonide (InSb) has attracted considerable attention over the last several years [2]-[4] and others. Its most interesting properties are the very low electron effective mass and high mobility. This property enables InSb to be used frequently in high speed applications in transistors and other devices [5]. Another important property of InSb is that it has a $0.17 \mathrm{eV}$ bandgap for bulk material at $300 \mathrm{k}$ which is considered the smallest bandgap among other binary III-V semiconductors. Consequently, InSb has been widely used in infrared detectors and filters [6][7].

The methods used to grow InSb and III-V semiconductors thin films, typically Molecular Beam Epitaxy (MBE) and Metal Organic Chemical Vapor Deposition (MOCVD) are expensive with low throughput. These techniques also require lattice matching. The chemical involved with MOCVD processing are also highly toxic [6]. Another more flexible technique is sputtering deposition. However, sputtering deposition produces amorphous InSb thin films, it is a costeffective technique. Fortunately, amorphous thin films can be recrystallized by annealing. In [8], InSb thin films were deposited by RF sputtering and annealed using Rapid Thermal

\footnotetext{
* Egyptian Armed Forces, Egypt; mohammad.awwad.eg@ieee.org

† Egyptian Armed Forces, Egypt; ahafiz@mailcity.com

* Professor, EAEA, ksharshar@yahoo.com
} 
Annealing (RTA) technique. While in [6] and [9], InSb thin films were RF sputtered on heated substrates.

In this paper, InSb thin films are deposited on glass and Si substrates by RF sputtering followed by $\mathrm{SiO}_{2}$ capping which is required to prevent evaporation of Antimony [10]. Then, the samples are vacuum annealed at various temperatures. The effects of annealing temperature on the films will be discussed with respect to crystal properties.

\section{Experiment}

A DSC analysis was performed using TA Q20 in a nitrogen gas environment for exploring the thermal behavior of InSb films. The temperature of $2.5 \mathrm{mg}$ of amorphous $\mathrm{InSb}$ is ramped from $40{ }^{\circ} \mathrm{C}$ to $550{ }^{\circ} \mathrm{C}$ at a rate of $10{ }^{\circ} \mathrm{C} / \mathrm{min}$.

Films of InSb are prepared by RF sputtering pumped to a base pressure of $5 \times 10^{-6}$ Torr by a turbomolecular pump to ensure low level of impurities. The gas used for sputtering is a highly pure Ar gas (99.9995\% pure). A 2 inch (100) Ge-doped P-type InSb wafer was used for sputtering the InSb films, while the oxide capping films were sputtered using 2 inche $99.995 \%$ purity $\mathrm{SiO}_{2}$ target. The InSb films were deposited on $\mathrm{Si}$ (111) and glass substrates. The resistivity of Si substrate at room temperature is between $35 \Omega \cdot \mathrm{cm}$ and $45 \Omega \cdot \mathrm{cm}$ with a $250 \mu \mathrm{m}$ thickness. InSb Films have been deposited by RF power of $40 \mathrm{~W}$ for 5 minutes on the silicon and glass substrates at room temperature, after the targets had been cleaned by pre-sputtering at $40 \mathrm{~W}$. The $\mathrm{SiO}_{2}$ capping films have been deposited by RF power of $100 \mathrm{~W}$ for 15 minutes. The capping layer role is to prevent evaporation of $\mathrm{Sb}$ from the InSb during furnace annealing, since elements of group $\mathrm{V}$ in the periodic table have relatively high partial pressure. In all sputtering processes, the substrates were rotated at maximum rate to obtain good uniformity. After sputtering processes, we will have 2 types of samples as depicted in Fig. 1.

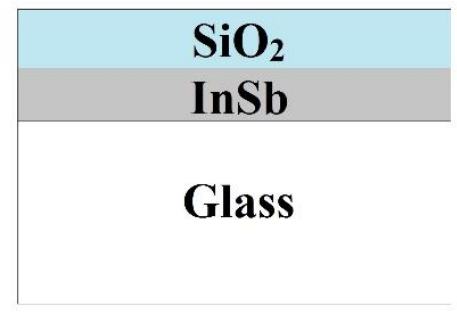

(a)

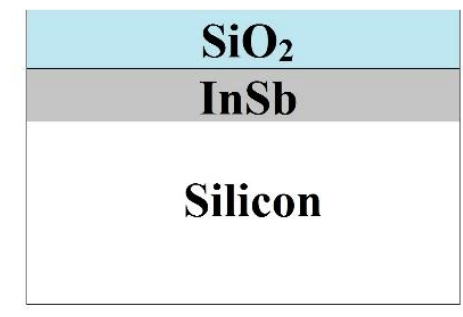

(b)

Fig. 1 Structure of the InSb film samples: (a) Capped InSb on glass substrate. (b) Capped InSb on Si substrate.

To study the effect of annealing temperature, 4 samples of glass substrates, represented in Fig. 1.a, have been annealed in vacuum furnace at $0.08 \mathrm{MPa}$ vacuum gauge pressure and temperatures of $150,300,390$, and $450{ }^{\circ} \mathrm{C}$ for 30 minutes. Also, 4 samples of (111) silicon substrate, represented in Fig. 1.b, have been annealed at $0.08 \mathrm{MPa}$ vacuum gauge pressure and temperatures of $150,300,390$ and $450{ }^{\circ} \mathrm{C}$ for 30 minutes to study the effect of annealing temperature on the bandgap of the thin film.

The structure of the films are characterized by X-ray diffraction (XRD) using Shimadzu XRD$6000\left(\mathrm{Cu}\right.$ Ka radiation, $\left.\lambda=1.5406^{\circ} \mathrm{A}\right)$. The scanning range is from $20^{\circ}$ to $80^{\circ}$ for samples with glass substrates. 
Fourier Transform infrared spectroscopy (FTIR) measurement has been carried out by JASCO FT/IR 4100 in absorption mode at room temperature in the spectral range between 2.5 and 9 $\mu \mathrm{m}$ while using the $\mathrm{SiO}_{2} / \mathrm{Si}$ wafer as reference.

\section{Results and Discussion}

Fig. 2 illustrates DSC measurement of InSb. The glass transition temperature $\left(\mathrm{T}_{\mathrm{g}}\right)$ appears around $200{ }^{\circ} \mathrm{C}$. The exothermic peak at a temperature of $390.9{ }^{\circ} \mathrm{C}$ indicates crystallization with a heat capacity of $3.37 \mathrm{~J} / \mathrm{g}$. While the endothermic peak $514.2{ }^{\circ} \mathrm{C}$ indicates the melting point of InSb. Although, the measured melting point of $514.2{ }^{\circ} \mathrm{C}$ is not very close to the previously reported value in [11] $\left(527^{\circ} \mathrm{C}\right)$, this shift is explainable since there might be a slight difference in composition due to germanium doping and/or sample preparation method.

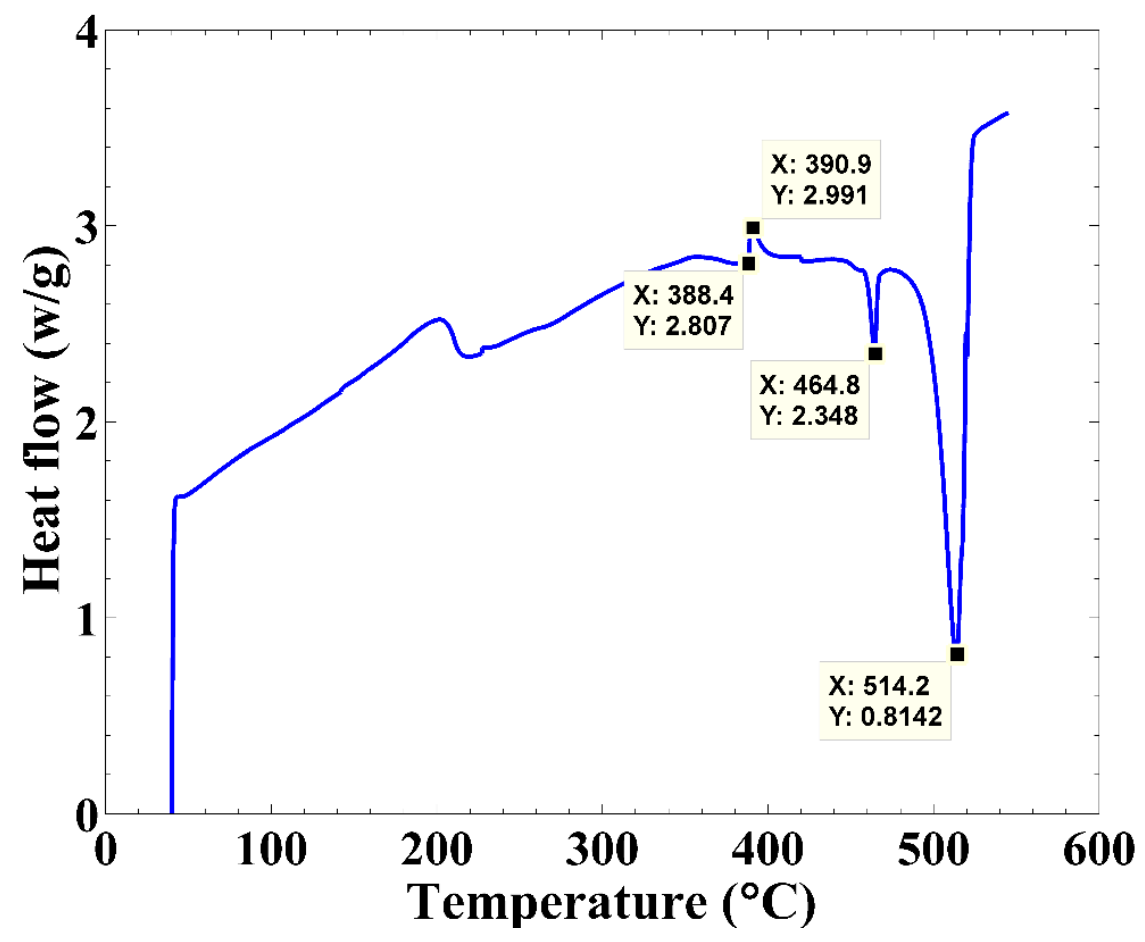

Fig. 2 DSC measurement of InSb

XRD patterns of the four samples annealed in vacuum furnace on glass substrates for 30 minutes at $150,300,390$, and $450{ }^{\circ} \mathrm{C}$ are plotted in Fig. 3. The XRD spectra show that the sample annealed at $150{ }^{\circ} \mathrm{C}$ is nearly amorphous, while the samples annealed at $300{ }^{\circ} \mathrm{C}$ and above are observed to be polycrystalline with ascending degrees of crystallinity. Diffraction peaks which assigned to be (111), (220), and (311) orientations of InSb with a zinc-blend structure at $2 \theta$ values of $23.73^{\circ}, 39.2^{\circ}$, and $46.32^{\circ}$. These values are very close to previous works [6], [8], [10], [12], [13].

Also, it is clear that the intensity of InSb (111) peak is larger than the other related peaks annealed at the same temperature. The intensity of $\mathrm{InSb}$ (111) peak of the sample annealed at $450{ }^{\circ} \mathrm{C}$ is greater than its counterpart annealed at $390{ }^{\circ} \mathrm{C}$ which in turn is greater than that annealed at $300{ }^{\circ} \mathrm{C}$. The sample annealed at $450{ }^{\circ} \mathrm{C}$ shows an observable single crystalline-like feature, and the other InSb-related peaks are very weak. According to equation (1) [14]

$$
\alpha(111)=\frac{I(111)}{I(111)+I(220)+I(311)} \times 100 \%
$$




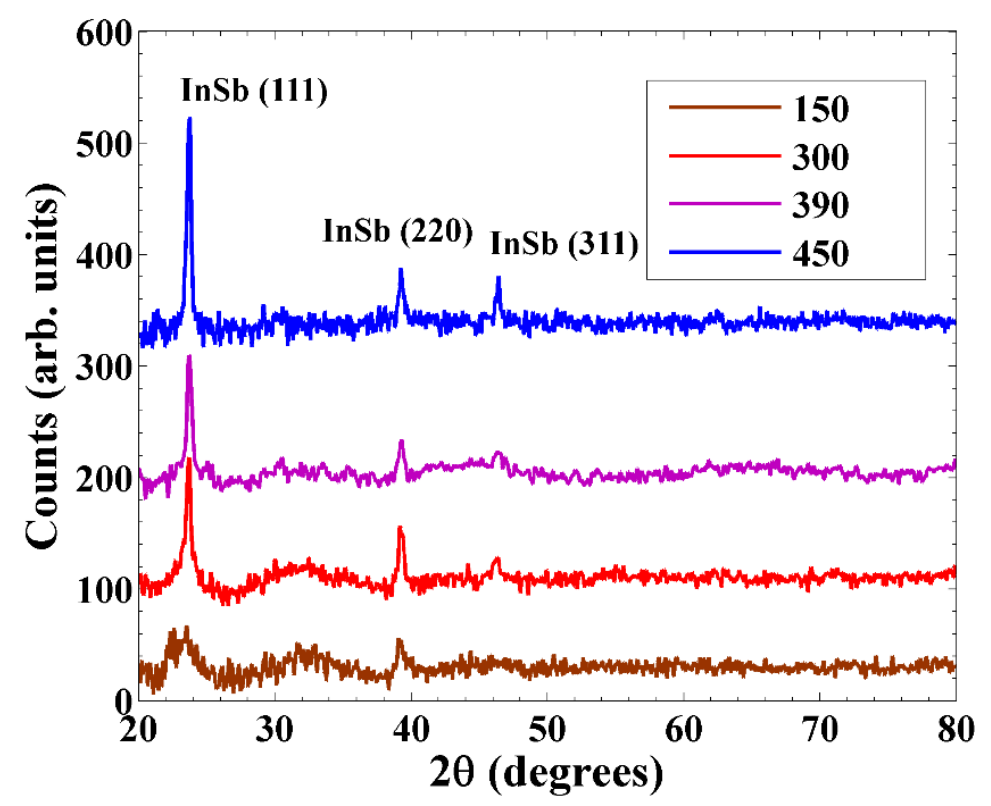

Fig. 3 XRD patterns of 4 InSb thin film samples annealed at different temperatures

where $\alpha$ is the orientation degree, and $\mathrm{I}(111), \mathrm{I}(220)$, and $\mathrm{I}(311)$ are the intensities of peaks (111), (220), and (311) respectively. It is found that the orientation degree $\alpha$ of peak (111) of InSb film annealed at $300{ }^{\circ} \mathrm{C}$ by vacuum furnace is about $51.6 \%$. However, as the temperature increases to $390{ }^{\circ} \mathrm{C}$, the orientation degree $\alpha$ of peak (111) increases to about $56.5 \%$. The orientation degree $\alpha$ of peak (111) is $61.5 \%$ after being recrystallized at $450{ }^{\circ} \mathrm{C}$. These results make InSb (111) a preferred orientation for the annealed samples, which agrees with results reported in [15]. This result indicates that the increase of annealing temperature leads to improvement of the crystalline quality of InSb film.

Fig. 4 illustrates the variations of grain size and full width at half maximum (FWHM) of annealed InSb films with temperature. Each XRD peak is carefully measured, and the grain size is calculated by the Scherrer formula [16],

$$
D=0.9 \lambda / B \cos \theta
$$

where $\lambda$ is the wavelength of the $\mathrm{X}$-ray radiation $(\lambda=0.154 \mathrm{~nm}), \mathrm{B}$ is the full width at half maximum (FWHM) of the peak (in radians) corrected for instrumental broadening, $\theta$ is the Bragg angle and $\mathrm{D}$ is the crystallite size. The average grain sizes were 16.2, 26.4, $30.5 \mathrm{~nm}$ for InSb thin films annealed at $300^{\circ}, 390^{\circ}$ and $450^{\circ}$, respectively. These average grain sizes are

larger than those annealed by RTA in [8], [12]. The average grain size of InSb film increases as the annealing temperature increases, because enhanced crystallinity of the film reduces the FWHM of the (111) peak. This represents a strong evidence for the possibility of obtaining high degree of crystallinity by annealing the as deposited InSb films.

Fig. 5 illustrates the measurement of infrared absorbance of $4 \mathrm{InSb}$ thin film samples on $\mathrm{Si}$ substrate using FTIR Spectroscopy in the wavelength range 2.5 to $9 \mu \mathrm{m}$. The 4 samples were annealed at $150,300,390$, and $450{ }^{\circ} \mathrm{C}$ for 30 minutes. It is clear that the absorption decreases with increasing the annealing temperature. In [17], it is shown that GaAs thin films had a similar behavior. The optical band gap energy of the film can be calculated using the Tauc model [18] in the high absorbance region, 


$$
(\alpha h v)^{2}=D\left(h v-E_{g}\right)^{n}
$$

where hv is the photon energy, Eg is the optical bandgap, and D is a constant; it can be found that $n=2$ corresponds to the indirect transition-type semiconductor and $n=1 / 2$ leads to the direct transition type [19]. After curve fitting the linear part of the Tauc plot as depicted in Fig. 6 , we can get the optical bandgap of InSb thin films annealed at different temperatures from the absorption spectrum. The optical bandgaps shift from 0.231 to 0.241 e.v. upon increasing the temperature from 150 to $450{ }^{\circ} \mathrm{C}$.

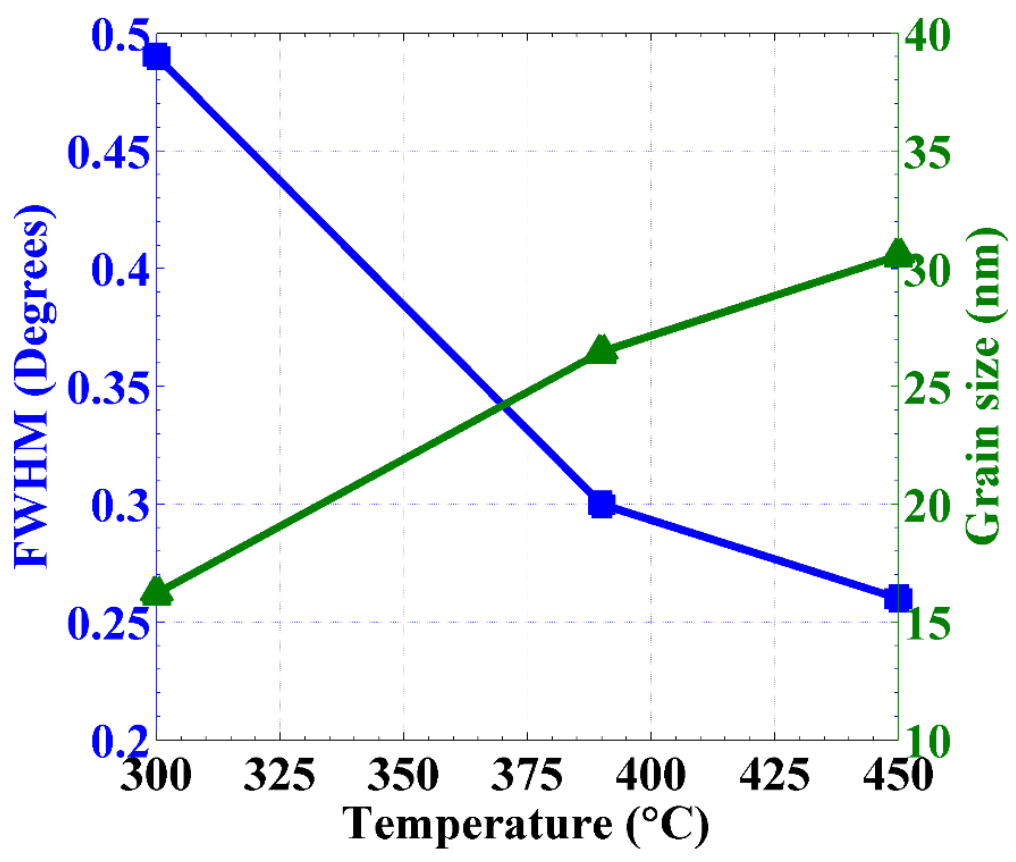

Fig. 4 The FWHM of the XRD and the grain size relation with the annealing temperature

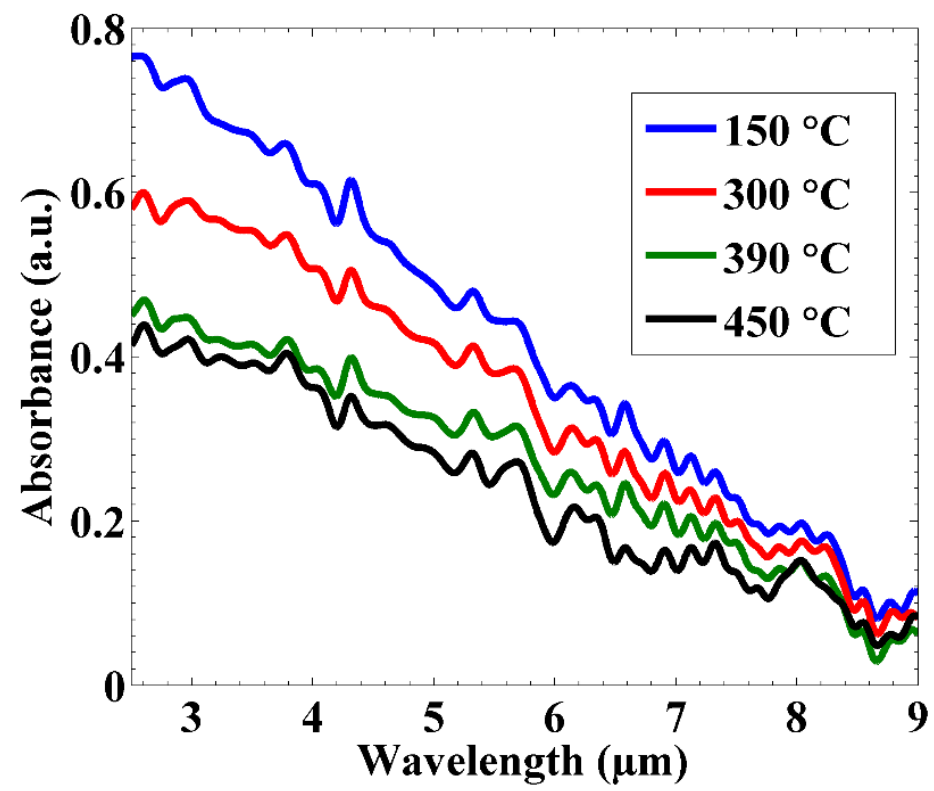

Fig. 5 Absorbance of InSb films annealed at different temperatures versus wavelength 


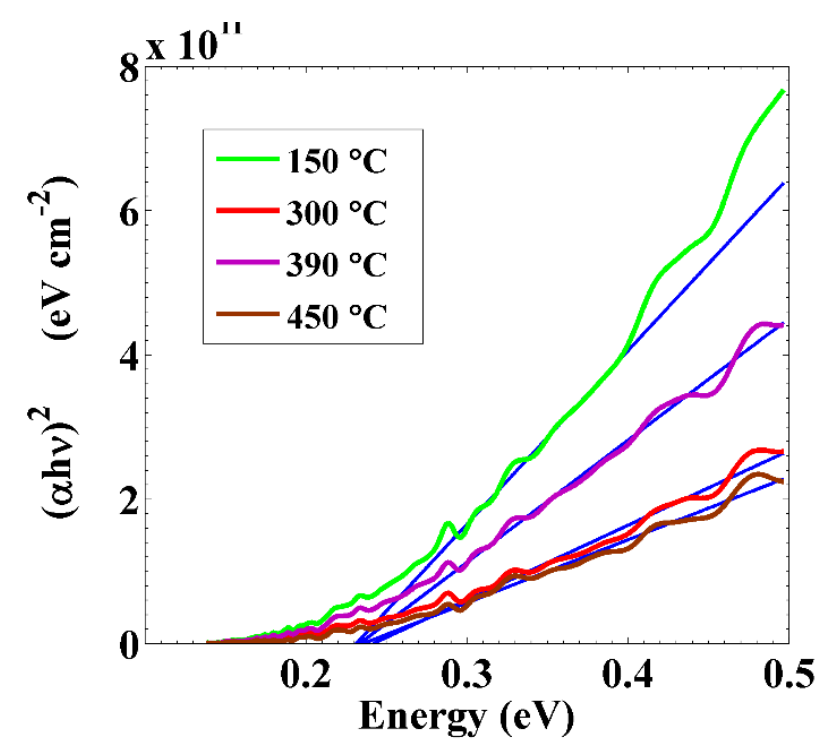

Fig. 6 Curves of ( $\alpha \mathrm{hv}) 2$ versus photon energy of InSb thin films annealed at different temperatures

\section{Conclusion}

InSb films have been deposited by RF sputtering technique. The DSC test showed that amorphous InSb films crystallizes at a temperature of $390{ }^{\circ} \mathrm{C}$ or above. The films have been annealed at various temperatures onto different substrates (glass and $\mathrm{Si}$ ). XRD peaks at (111), (220) and (311) planes were shown, which indicates the formation of crystalline grains. On increasing the annealing temperature, the orientation degree increases, the average grain size increases to $30 \mathrm{~nm}$, and the crystalline quality of $\mathrm{InSb}$ film improves. FTIR spectroscopy indicates the decrease of optical transmittance on increasing the annealing temperature, the analysis shows that InSb thin films have bandgaps between 0.233 and 0.241 e.v. It can be concluded that as deposited InSb films can show high degree of crystallinity using proper annealing treatment.

\section{Acknowledgments}

We would like to thank our colleagues from the Military Technical Research Center especially Dr. Tamer Roshdy and Dr. Mahmoud Anwar Saleh who provided resources and expertise that greatly assisted the research.

We thank Dr. Lobna Aly Abd El Wahab, Head of Radiation Research Division, EAEA, for assistance with DSC technique and for comments that greatly improved the manuscript.

We would also like to show our gratitude to Dr. A.Baraka, Dr. M.Gobara, and M.Zorainy, Chemical Engineering Department, Military Technical College (MTC), for their great support and assistance in this research. 


\section{References}

[1] P. Bhattacharya, Semiconductor optoelectronic devices. Prentice Hall, 1994.

[2] V. Pusino et al., "InSb Photodiodes for Monolithic Active Focal Plane Arrays on GaAs Substrates," IEEE Trans. Electron Devices, vol. 63, no. 8, pp. 3135-3142, 2016.

[3] J. P. Perez, A. Evirgen, P. Christol, A. Cordat, A. Nedelcu, and J. Abautret, "Midwave infrared InSb nBn photodetector," Electron. Lett., vol. 50, no. 20, pp. 1472-1473, 2014.

[4] L. Hoglund et al., "Minority Carrier Lifetimes in InSb/InAsSb Quantum Dot and InAsSb nBn Photodetectors," IEEE Photonics Technol. Lett., vol. 27, no. 23, pp. 2492-2495, 2015.

[5] T. Ashley et al., "Heterogeneous InSb quantum well transistors on silicon for ultra-high speed, low power logic applications," Electron. Lett., vol. 43, no. 14, p. 777, 2007.

[6] T. Zens, P. Becla, A. M. Agarwal, L. C. Kimerling, and A. Drehman, "Amorphous InSb and InAs ${ }_{0.3} \mathrm{Sb}_{0.7}$ for long wavelength infrared detection," in SPIE 8012, Infrared Technology and Applications XXXVII, 2011, vol. 8012, p. 80123Y.

[7] M. Singh and Y. K. Vijay, "Electrical, optical and structural properties of indiumantimonide (In-Sb) bilayer film structure," Indian J. Pure Appl. Phys., vol. 42, no. 8, pp. 610-614, 2004.

[8] D.-Y. Li, H.-T. Li, H.-H. Sun, and L.-C. Zhao, "Effects of rapid thermal annealing on the morphology and optical property of ultrathin $\mathrm{InSb}$ film deposited on $\mathrm{SiO} 2 / \mathrm{Si}$ substrate," Chinese Phys. B, vol. 22, no. 2, p. 27802, 2013.

[9] T. Miyazaki, M. Kunugi, Y. Kitamura, and S. Adachi, "Epitaxial growth of InSb films by r.f. magnetron sputtering," Thin Solid Films, vol. 287, no. 1-2, pp. 51-56, 1996.

[10] M. Tomisu, N. Inoue, and Y. Yasuoka, "Annealing effect of vacuum evaporated InSb thin films," Vacuum, vol. 47, no. 3, pp. 239-242, 1996.

[11] M. Levinshtein, S. Rumyantsev, and M. Shur, Handbook Series on Semiconductor Parameters, vol. 1. WORLD SCIENTIFIC, 1996.

[12] D. Li, H. Li, H. Sun, and L. Zhao, "Characterization of ultrathin InSb nanocrystals film deposited on SiO2/Si substrate.," Nanoscale Res. Lett., vol. 6, no. 1, p. 601, 2011.

[13] X. Cai and J. Wei, "Temperature dependence of the thermal properties of InSb materials used in data storage," J. Appl. Phys., vol. 114, no. 8, pp. 1-8, 2013.

[14] P. Taylor, "Ferroelectric and Domain Properties of," Ferroelectrics, vol. 357, no. 919435512, pp. 259-263, Sep. 2010.

[15] J. Pelleg, L. Z. Zevin, S. Lungo, and N. Croitoru, "Reactive-sputter-deposited TiN films on glass substrates," Thin Solid Films, vol. 197, no. 1-2, pp. 117-128, 1991.

[16] C. C. Koch, I. A. Ovid'ko, S. Seal, and S. Veprek, Structural Nanocrystalline Materials: Fundamentals and Applications. 2007.

[17] R. A. Abdullah, K. A. Adem, and M. A. Razooqi, "The Optical Properties of GaAs Thin films Prepared by Flash Evaporation Technique," 2008.

[18] J. Tauc, “Amorphous and Liquid Semiconductors," Opt. Acta Int. J. Opt., vol. 17, no. 12, pp. 952-952, 1974.

[19] S. Ohkubo, K. Aoki, and D. Eto, "Temperature dependence of optical constants for InSb films including molten phases,” Appl. Phys. Lett., vol. 92, no. 1, p. 11919, 2008. 